\title{
Aplicaciones de la neurofisiología cognitiva y la estimulación cerebral no invasiva al estudio del lenguaje
}

\author{
JAVIER J. GoNZÁLEZ-RosA \\ Universidad de Cádiz \\ Departamento de Psicología \\ Facultad de Ciencias de la Educación \\ Campus Universitario Río San Pedro \\ Avda. República Saharaui s/n \\ 11510 - Puerto Real \\ Email: javier.rosa@uca.es
}

\begin{abstract}
APLICACIONES DE LA NEUROFISIOLOGÍA COGNITIVA Y LA ESTIMULACIÓN CEREBRAL NO INVASIVA AL ESTUDIO DEL LENGUAJE
\end{abstract}

\begin{abstract}
RESUMEN: Los potenciales relacionados con eventos (ERP) son un método de evaluación y de mapeo cerebral que permite entender la dinámica temporal del procesamiento de la información, dotando a la neurociencia de una herramienta de gran valor para escudriñar el procesamiento del lenguaje en niños y adultos, asi como en trastornos cerebrales. Su uso está permitiendo comprender con precisión aquellos marcadores cerebrales involucrados en aspectos tan importantes como la adquisición del lenguaje o la integración y los desajustes semánticos y sintácticos. Por otra parte, el empleo de técnicas de estimulación cerebral no inuasiuas está suponiendo una auténtica revolución en el estudio del lenguaje; es una potente herramienta para la evaluación de aquellas redes cerebrales que lo facilitan o lo inhiben y permite mejorar o corregir sustancialmente el aprendizaje y el rendimiento en pruebas de lectura y comprensión o recuerdo, tanto en población sana como en tratamientos rehabilitadores en población clínica.
\end{abstract}

PALABRAS CLAVES: Electroencefalografia; Potenciales relacionados con eventos; Lenguaje; Afasia; Estimulación cerebral transcraneal.

SUMARIO: 1. Neurofisiología cognitiva. 1.1. Electrofisiología (EEG) y Potenciales Relacionados con Eventos (ERP). 1.2. Significado funcional de los ERP. 1.3. El estudio de lenguaje mediante ERP. 1.4. Ejemplos de in vestigaciones de ERP y lenguaje. 2. Estimulación cerebral no invasiva al estudio del lenguaje. 2.1. Estimulación cerebral no invasiva. 2.2. Estimulando el lenguaje: estimulación transcraneal por corriente continua (DCS). 2.3. Estimulando el lenguaje: estimulación magnética transcraneal (TMS) 2.4. Interpretación de los resultados obtenidos con TMS/tDCS después de una tarea de lenguaje (cognitiva).

Fecha de Recepción

Fecha de Revisión

Fecha de Aceptación

Fecha de Publicación

\section{APPLICATIONS OF COGNITIVE NEUROPHYSIOLOGY AND NON- INVASIVE BRAIN STIMULATION TO THE STUDY OF LANGUAGE}

ABSTRACT: Event-Related Potentials (ERP) is a method of brain assessment and mapping that allows understanding the temporal dynamics of information processing, providing neuroscience with a valuable tool for studying language processing in children and adults, as well as brain disorders associated to language disorders. It improves the understanding of those brain markers involved in aspects as language aoquisition or how the semantic and syntactic level cooperate. On the other hand, the use of non-invasive brain stimulation techniques is representing a revolution in the study of language: it is a powerful tool for the evaluation of brain networks and it allows us to improve or correct learning and performance in reading and comprehension or memory tests, both in healthy and clinical populations.

KEY WORDS: Event-Related Potentials; Language; Aphasia; Brain stimulation; Electroencephalography.

SUMMARY: 1 Cognitive neurophysiology. 1.1. Electrophysiology (EEG) and Event Related Potentials (ERP). 1.2. Functional meaning of ERP. 1.3. The study of language using ERP. 1.4. Examples of ERP and language research. 2. Non-invasive brain stimulation to the study of language. 2.1. Noninvasive brain stimulation. 2.2. Stimulating language: transcranial direct current stimulation (tDCS). 2.3. Stimulating language: transcranial magnetic stimulation (TMS). 2.3.1. The use TMS in the study of language 2.4. Interpretation of the results obtained with TMS/tDCS after a language (oognitive) task.
APPLICATIONS DE LA NEUROPHYSIOLOGIE COGNITIVE ET DE LA STIMULATION CEREBRALE NON INVASIVE A L'ETUDE DU LANGAGE

RÉSUMÉ : Les potentiels liés aux événements (ERP) sont une méthode d'évaluation et de cartographie du cerveau qui nous permet de comprendre la dynamique temporelle du traitement de linformation, fournissant aux neurosciences un outil précieux pour examiner le traitement du langage chez les enfants et les adultes, ainsi que dans les troubles cérébraux où il y a un certain trouble du langage. Son utilisation nous permet de comprendre avec précision les marqueurs cérébraux impliqués dans des aspects aussi importants que l'acquisition ou lintégration du langage et les inadéquations sémantiques et syntaxiques. D'autre part, l'utilisation des techniques de stimulation cérébrale non invasives représente une véritable révolution dans l'étude du langage, non seulement parce que nous disposons dun outil puissant pour lévaluation des réseaux cérébraux qui le facilitent ou linhibent, mais aussi parce qu'elle nous permet d'améliorer ou de corriger de manière substantielle lapprentissage et les performances dans les tests de lecture et de compréhension ou de mémoire, tant dans les populations saines que dans les traitements de réadaptation dans les populations cliniques.

MOTS CLÉS : Potentiels liés aux événements ; Langue ; Aphasie ; Stimulation du cerveau ; Électroencéphalographie.

SOMMAIRE : 1 . Neurophysiologie ognitive. 1.1. Électrophysiologie (EEG) et potentiels liés aux événements (ERP). 1.2. Signification fonctionnelle de l'ERP. 1.3. Létude des langues àlaide de l'ERP. 1.4. Exemples de recherche en matière d'ERP et de langues. 2. Stimulation oérébrale non invasive pour létude du langage. 2.1. Stimulation cérébrale non invasive. 2.2 . Stimulation du langage : stimulation transcrânienne par courantcontinu (tDCS). 2.3. Stimuler le langage : la stimulation magnétique transcrânienne (TMS). 2.4. Interprétation des résultats obtenus avec le TMS / tDCS après une tâche linguistique (oognitive). 


\title{
Aplicaciones de la neurofisiología cognitiva y la estimulación cerebral no invasiva al estudio del lenguaje
}

\author{
FLORENCIA SANMARTINO \& JAVIER J. GONZÁLEZ-ROSA
}

\section{INTRODUCCIÓN}

El Grupo de Neuroimagen y Psicofisiologia (GNP: IN-CO12), integrado en el Instituto de Investigación e Innovación Biomédica de Cádiz (INiBICA) y en la Universidad de Cádiz, es un grupo de investigación integrado por varios investigadores que, en estrecha colaboración con otros profesionales clínicos neurólogos y neurocirujanos del Hospital Universitario Puerta del Mar, está actualmente llevando a cabo diferentes líneas de investigación centradas principalmente en el uso de técnicas de mapeo cerebral (e.g., EEG, fMRI) y de estimulación cerebral no invasiva (e.g., TMS, tSMS) e invasiva (DBS), para poder así explorar las bases neurales de la cognición humana, con un especial interés en la evaluación de las alteraciones cognitivas en pacientes neurológicos y psiquiátricos.

En la actualidad, todas las líneas de investigación y las distintas metodologías de análisis empleadas quedan enmarcadas dentro de tres proyectos de investigación que han sido recientemente financiados por el Ministerio de Ciencia e Innovación, el Ministerio de Economía y Competitividad (RYC-2015-18467; RTI2018-096951-AI00; PSI2017-85951-R; EQC2018-004728-P; EQC2019-006401-P), y la Consejería de Salud de la Junta de Andalucía (PI-0025-2017; PI-0034-2019). Como nexo común, estos tres proyectos persiguen, en líneas generales, estudiar las redes corticosubcorticales que subyacen al deterioro cognitivo en pacientes neurológicos (e.g., Esclerosis Múltiple, Enfermedad de Parkinson, Migraña Crónica, e Ictus, etc.) y psiquiátricos (e.g., TOC, Esquizofrenia, etc.) mediante el uso de la integración multimodal de datos EEG y datos de resonancia magnética funcional (RMf), de datos EEG y estimulación magnética/eléctrica transcraneal, pero también mediante el desarrollo de nuevos enfoques de mapeo funcional, como el co-registro de EEG durante protocolos de estimulación cerebral profunda (DBS) o el registro simultáneo de datos intracranales y EEG desde regiones cerebrales profundas, con una finalidad terapéutica, en pacientes psiquiátricos y neurológicos tratados con DBS.

Más en detalle, las principales líneas de investigación pueden resumirse en los siguientes puntos:

\section{Cognición humana y deterioro cognitivo}

- Evaluación mediante técnicas electrofisiológicas y de neuroimagen de las bases neurales de los procesos cognitivos (e.g., funciones ejecutivas, atención, lenguaje); diseño de nuevas tareas cognitivas.

- Estudio de los mecanismos cerebrales que subyacen a la función cognitiva tanto en población sana como en pacientes con alteraciones neurológicas y psiquiátricas.

- Evaluación de los patrones de actividad cerebral y su relación con la presencia del deterioro cognitivo y alteraciones clínicas.

- En la actualidad, examen de pacientes con enfermedad de Parkinson, Esclerosis Múltiple, demencias, trastorno obsesivo-compulsivo, y migraña crónica.

\section{Métodos de imagen cerebral}

- EEG y ERP: Uso avanzado de análisis y técnicas electrofisiológicas; empleo de equipos de alta densidad de EEG para entender la dinámica espaciotemporal de 
activación de las redes corticales en reposo y durante la realización de tareas cognitivas, tanto en población sana como patológica.

- Nuevos análisis y soluciones metodológicas para la localización de generadores cerebrales mediante toolboxes como Analyzer, EEGLAb, Fieldtrip, SPM12, Brainstorm, etc.

- Análisis de datos de neuroimagen estructural (MR) y funcional (fMRI): cálculos de atrofia cerebral, carga lesional, medidas de volumetría regional, cortical thickness, etc.

- Fusión e integración multimodal de datos electrofisiológicos (EEG) y hemodinámicos (fMRI) adquiridos separada o simultáneamente.

- Registros intracraneales (icEEG) en humanos post-neurocirugía.

- Registros biométricos de actividad periférica con acelerómetros, EMG; EKG, actividad galvánica de la piel (GSR), y uso eye-tracker para controlar y estudiar los movimientos oculares durante la realización de tareas cognitivas.

\section{Neuromodulación}

- Empleo de distintas técnicas de neuromodulación como la estimulación magnética transcraneal (TMS), por corriente continua (tDCS), y por campos magnéticos estáticos (tSMS), en sujetos sanos y con trastornos neurológicos.

- Estimulación cerebral profunda (DBS) en pacientes neurológicos y psiquiátricos.

- Co-registros simultáneos de EEG durante protocolos de DBS.

\section{NEUROFISIOLOGÍA COGNITIVA}

\subsection{Electrofisiología (EEG) y Potenciales Relacionados con Eventos (ERP)}

Existen numerosas técnicas de neuroimagen que permiten con menor o mayor exactitud afrontar dos de los mayores desafios en el estudio del cerebro humano, como son, por una parte, poder medir la dinámica temporal de los procesos cognitivos, y por otra, localizar dónde ocurre o qué regiones cerebrales son responsables de dichos procesos. Entre estas técnicas, destaca por diversas razones la electroencefalografia (EEG), la cual consiste en el registro y evaluación de los potenciales eléctricos generados por el cerebro y obtenidos a través de electrodos situados sobre la superficie del cuero cabelludo (Niedermeyer y Lopez da Silva, 1999). Lo detectado por el EEG es el resultado de la actividad sumada de millones de potenciales postsinápticos excitadores e inhibidores de las neuronas piramidales de la corteza cerebral con similar orientación espacial. Así, la señal de EEG es el producto del registro de millones de neuronas, principalmente neuronas piramidales, que están disparando de menara sincronizada. El EEG está considerado una herramienta de enorme utilidad para medir de forma directa la actividad eléctrica generada desde el cerebro, siendo además un método no invasivo y con una alta resolución temporal, que abre la posibilidad para explorar la actividad neuronal y el funcionamiento del cerebro humano (Carretié e Iglesias, 2008). 
En el EEG se puede observar la actividad cerebral espectral (i.e., actividad oscilatoria) tanto espontánea como asociada a un evento, pero, además, podemos estudiar la respuesta del cerebro a estímulos o acontecimientos discretos ocurridos en el tiempo en forma de cambios en la amplitud del EEG (no en el espectro). Este registro se conoce como potenciales evocados o PE (del inglés EP: evoked potentials), los cuales se obtienen estimulando una vía sensorial determinada para obtener una respuesta fisiológica o correlato del procesamiento (sensorial, motor, afectivo, cognitivo) (Pizzagalli, 2007; Carretié e Iglesias, 2008). Así, los PE consisten en cambios en la actividad eléctrica del sistema nervioso central o de la corteza cerebral originados por la percepción de algún evento. En función de la modalidad sensorial mediante la cual se percibe el estímulo, evento o acontecimiento que produce dicho potencial, éstos se clasifican como potenciales evocados visuales, auditivos, somatosensoriales, etc. De igual modo, las respuestas motoras también elicitan señales eléctricas corticales que se pueden registrar, y que reciben el nombre de potenciales evocados motores.

En neurofisiología, existe una gran tradición en estudiar PE automáticos o de corta latencia, ya que ofrecen una valiosa información sobre la velocidad de conducción de las vías sensoriales y sobre la integridad funcional de estas. Desde un punto de vista cognitivo, sin embargo, pueden ser de mayor interés aquellos $\mathrm{PE}$ que se relacionan con aspectos cognitivos del procesamiento de la información, los llamados $\mathrm{PE}$ cognitivos o $\mathrm{PE}$ relacionados con eventos (event-related potentials -ERP). Éstos consisten en cambios en la actividad eléctrica de la corteza cerebral originados por la percepción de algún evento o acontecimiento discreto (por ejemplo, el sonido de un timbre, decidir mover la mano, discriminar entre dos estímulos con distintos colores, etc.) (Pizzagalli, 2007; Carretié e Iglesias, 2008).

Las señales psicofisiológicas relativamente complejas que constituyen los $\mathrm{PE}$ implican diversas inflexiones o componentes a lo largo del tiempo, y son diferentes en amplitud, polaridad y latencia. Estas ondas se denominan componentes de los PE o ERP y suelen identificarse: i) en función de su polaridad o carga eléctrica como positivas $(\mathrm{P})$ o negativas $(\mathrm{N})$; ii) en función de su latencia o momento temporal de aparición; y iii) por su topografia o distribución sobre el cuero cabelludo. Además de lo citado, los componentes de los PE también suelen ir definidos por una nomenclatura que indica el orden de aparición con relación a su latencia temporal (e.g., componente P1: positividad a los $100 \mathrm{mseg}$, componente N2: negatividad a los $200 \mathrm{mseg}$, componente P3: positividad a los $300 \mathrm{mseg}$, etc.) Los PE y ERP están formados, por tanto, por distintas inflexiones o componentes, cada uno de los cuales tendria una latencia, polaridad, topografia sobre el cuero cabelludo y un significado funcional distinto o relativamente bien delimitado. En general, se acepta que la latencia un componente representaría una medida objetiva de la velocidad del procesamiento de los estímulos en distintos estadios. Por su parte, la amplitud de los componentes se relacionaria con la cantidad de actividad cerebral necesaria en un proceso cognitivo concreto (Picton e Hillyard, 1988). 
Generalmente, en neurofisiología clinica, es común estudiar componentes de latencia muy corta, por debajo de los $100 \mathrm{mseg}$, también llamados PE "exógenos", los cuales tienen su origen neural en las vias cerebrales que llevan la información aferente desde los órganos sensoriales hasta las cortezas primarias, o bien en las vias eferentes que se dirigen desde la corteza motora a los músculos. Sin embargo, para el estudio de variables psicológicas y procesos cognitivos, tiene más interés el estudio de los componentes de los PE "endógenos" o ERP, los cuales tienen una latencia de aparición más lenta, por encima de los 100 mseg y hasta los 1000 mseg y más. Estos componentes tienen un origen cortical a nivel post-sináptico y se deben a cambios sincrónicos en la polarización de poblaciones neuronales relativamente grandes, aunque sus generadores neurales específicos son más inciertos. Los componentes endógenos reflejan, por tanto, el procesamiento de estímulos o eventos ya analizados por las cortezas primarias y que posteriormente son procesados en serie y/o en paralelo por las distintas cortezas asociativas y, finalmente, por las cortezas de integración polimodal (Picton e Hillyard, 1988; Bressler y Ding, 2006).

\subsection{SIGNIFICADO FUNCIONAL DE LOS ERP}

El análisis de la latencia de los distintos componentes (cognitivos) de los ERP hacen que sea posible identificar distintos aspectos del procesamiento de la información que están retrasados o prolongados, por ejemplo, debido a un proceso cognitivo más tardío, o a un trastorno neurológico. Estas latencias pueden proporcionar una medida más exacta del curso temporal de los acontecimientos neurales que están interviniendo en el transcurso de una tarea, lo que no se puede inferir con tanta precisión usando test neuropsicológicos o menos aún con otras pruebas de neuroimagen. Si una lesión cerebral induce un enlentecimiento en un estadio o etapa particular del procesamiento, la latencia de un componente de los $\mathrm{PE}$, y cada componente que le sigue, pueden estar retrasados. Así, identificando qué componentes obtienen latencias normales y cuáles están retrasados, podemos identificar el locus neural del procesamiento retrasado o en el que ocurre un determinado déficit, pero también podriamos identificar en qué momento percibimos un estímulo y cuando pasa a la memoria de trabajo o es, por el contrario, ignorado.

Aunque existen numerosos estudios realmente significativos sobre los ERP más tempranos (P1, N1, P2) o sobre otros ERP mucho más tardíos en cuanto a su implicación en el procesamiento de la información (N400, P600), la mayoría de los estudios cognitivos en lo que se usa metodología ERP evalúan sobre todo el componente N2 y el P3 (Bressler y Ding, 2006; Pizzagalli, 2007). Cuando se habla del componente N2, se suele hablar del llamado "complejo N2", ya que existen distintos subcomponentes en torno a esa latencia y polaridad que varian en su distribución topográfica y en su significado funcional, y que son dependientes también de la tarea que se realice. 
En la modalidad auditiva suele ser muy estudiada la llamada mismatch negativity o MMN, la cual se define como una respuesta eléctrica de polaridad negativa para discriminar cualquier cambio (desviante) en una secuencia repetitiva (estándar) de estimulación, generalmente, auditiva. Suele obtenerse sustrayendo en el registro de los ERP la condición desviante menos la estándar y en tareas o paradigmas de tipo Oddball. Básicamente, estas tareas suelen consistir en ofrecer dos tipos de estímulos, uno frecuente y con una alta frecuencia de aparición, y otro infrecuente o desviante y con una menor frecuencia de aparición (Näätänen, 2003). Típicamente, las tareas tipo Oddball suelen provocar dos respuestas cerebrales eléctricas o dos componentes característicos como respuesta, el componente MMN y el componente P3.

El componente MMN tiene una gran relevancia clinica y experimental debido a que puede aparecer incluso en ausencia de atención, lo que lo convierte en una herramienta muy útil desde un punto de vista clínico para comprobar el estado de la función cerebral en pacientes poco colaborativos o en niños. La aparición de esta inflexión llamada MMN está basada en la presencia de una traza o huella de memoria que representaria exactamente aspectos relacionados con las características de los estímulos auditivos, como su frecuencia o secuencia temporal, así como de las secuencias estimulares. Si en esta huella de memoria aparece un estímulo desviante o infrecuente, entonces el proceso de la detección del cambio ocurre y genera el componente MMN. Numerosos estudios han determinado que los generadores neurales de la MMN se localizarian en diferentes regiones del córtex auditivo, con importantes contribuciones del tálamo y del hipocampo. Además, se ha sugerido que el lóbulo frontal izquierdo también participaría activamente involucrándose en el cambio atencional ante la novedad (Näätänen, 2003; Bressler y Ding, 2006).

Generalmente, el significado funcional del componente MMN podría resumirse como "una respuesta cerebral a las violaciones de una regla, establecida por una secuencia de estímulos sensoriales". Permite discriminar cualquier cambio (desviante) en una secuencia repetitiva (estándar) de estimulación auditiva.

Por su parte, el componente P3 ha sido la medida psicofisiológica que más atención investigadora ha recibido en las últimas décadas. Hay un acuerdo general en que el P3 o P300 no sería un potencial cerebral unitario, ya que representaría la suma de la actividad de varias áreas neurales ampliamente distribuidas, representando en particular la integración de áreas neurales de asociación. Está también generalmente aceptado que podemos diferenciar en P3 dos subcomponentes, el P3a, de distribución más anterior o frontal, y el P3b, con una distribución más posterior o parietal (Polich y Herbst, 2000; Pizzagalli, 2007). E1 P3 es el siguiente componente genuino que se forma tras el componente N2 o MMN, durante tareas de tipo Oddball (aunque también en muchas otras tareas). Se observa una vez se ha detectado el cambio o la novedad en la estimulación. Es por ello por lo que se suele definir como una respuesta de orientación cerebral ante un estímulo 
infrecuente o una novedad en la secuencia estimular. Suele aparecer bien ante estimulos novedosos que no son objetivo de la tarea (distribución más sobre electrodos frontales), y que principalmente reflejan cambios involuntarios de atención relacionados con los cambios en el ambiente, bien ante estímulos que implican acceso a los procesos de memoria y que son evocados por la evaluación de los estímulos que requieren algún tipo de acción. Los generadores neurales del P3 estarian formados por la suma de una amplia red de fuentes neurales, ya que este componente representaría la actividad de múltiples áreas, destacándose importantes contribuciones del hipocampo, áreas parahipocampales, lóbulo temporal, y del lóbulo frontal (Polich y Herbst, 2000; Bressler y Ding, 2006).

La latencia del P3 se ha relacionado con la cronometría mental o un indice del tiempo de procesamiento requerido antes de la generación de la respuesta, por lo que es una medida temporal sensible a la actividad neural subyacente a los procesos que implican a la atención y la memoria inmediata. En el caso del componente P3, la amplitud seria un índice de la actividad cerebral requerida en el mantenimiento de la memoria de trabajo. Además, la amplitud del P3 sería igualmente proporcional a la cantidad de recursos atencionales dedicados en una tarea dada. Se asume que la variación en la amplitud del P3 reflejaría, por tanto, el grado o calidad con la que es procesada la información (Picton y Hillyard, 1988; Carretié e Iglesias, 2008).

\subsection{EL ESTUDIO DEL LENGUAJE MEDIANTE ERP}

Las ventajas metodológicas de los ERP han dado como resultado un número cada vez mayor de estudios ERP en neurociencia cognitiva, psicología cognitiva, neuropsicologia, neurología psicolingüística y neurolingüística.

Debido a las particularidades que tiene el estudio del lenguaje humano, el empleo de la metodología de los ERP ha sido capital para comprender mejor la dinámica temporal del procesamiento de la información, dotando a la neurociencia de una potente herramienta para escudriñar el procesamiento del lenguaje en niños y adultos, así como el procesamiento normal del lenguaje y en trastornos cerebrales donde hay alguna alteración del lenguaje. De esta manera, disponemos de una excelente herramienta para evaluar los aspectos neuro-cronométricos del procesamiento del lenguaje. Muchos estudios que utilizan esta metodología han vinculado las respuestas específicas de los ERP a diferentes tipos de procesos lingüísticos en diferentes idiomas.

Así, usando cualquier paradigma o tarea computerizada adaptada al registro simultaneo de actividad EEG, la mayoria de los estudios han logrado descifrar en buena medida cómo nuestro cerebro procesa la información relacionada con el lenguaje. Por ejemplo, ante una tarea de lenguaje auditiva, es habitual que encontremos entre los ERP el componente N1 (relacionado con el procesamiento perceptual pre-atencional en cortezas primarias), el componente P2 (procesamiento perceptual pre-atencional en cortezas se- 
undarias y detección del estímulo), el componente N2 (detección del estímulo e integración de las características del estímulo), el componente P3 (categorización de estímulos y actualización de memoria), N400 (procesamiento semántico y conceptual), y el componente P600 (procesamiento sintáctico). Es importante aquí destacar que, como suele ocurrir en procesos cognitivos superiores en el ser humano, como son la memoria o el lenguaje, existen una serie de componentes de los ERP que pueden ser específico en el estudio del lenguaje.

\subsection{EJEMPLOS DE INVESTIGACIONES DE ERP Y LENGUAJE}

Ya a principios de los años 80, Kutas e Hillyard (1980) fueron los primeros en informar sobre un componente de los ERP que estaba estrechamente relacionado con el procesamiento del lenguaje. Ellos descubrieron que palabras semánticamente erróneas en el contexto de una frase elicitaban una negatividad con topografia centro-parietal aproximadamente a los $400 \mathrm{~ms}$ desde la aparición de dicho estímulo, por lo que fue nombrada como N400 (Kutas e Hillyard, 1980; 1984). Posteriormente, son muchísimas las investigaciones que han demostrado que la amplitud del N400 aumenta junto con las dificultades léxico-semánticas en una variedad de contextos, como pueden ser una sola palabra, lista de palabras, una oración o la totalidad de un discurso. En general, se cree que las modulaciones de la amplitud N400 reflejarían los costes de procesamiento de la integración del significado de una palabra en la representación del significado general que se construye sobre la base de la entrada léxica en cuestión. Otros autores proponen que el N400 reflejara también la facilidad con la que se puede acceder a la información desde la memoria semántica. Las palabras que son semánticamente incongruentes o tienen un pobre ajuste semántico dado el contexto anterior provocan igualmente un N400 de mayor amplitud que las palabras que se ajustan bien dentro de su contexto. En cualquier caso, existe la opinión generalizada que el N400 refleja la integración semántica de una palabra en su contexto más amplio (Kutas y Federmeier, 2000; Friederici, 2002).

En el dominio de la sintaxis se modularian, en cambio, un grupo diferente de respuestas o componentes de los ERP. Así, existen dos componentes que son los frecuentemente descritos en este sentido, la left-anterior negativity (LAN), entre los 200 y 500 ms tras el inicio del estimulo (Friederici et al., 1993; Gunter et al., 2000), y el P600, que ocurre más tarde, entre los 500$900 \mathrm{~ms}$, sobre electrodos centro-parietales (Neville et al., 1991; Osterhout et al., 1994). Existe un gran debate desde hace décadas sobre en qué medida estos componentes reflejarian procesos sintácticos específicos (Osterhout et al., 1994; Friederici et al., 2002) o más bien mecanismos cognitivos de dominio general (Coulson et al., 1998). Sin embargo, parece haber una consistencia considerable en las condiciones que provocan las negatividades anteriores y/o los efectos de P600. Mientras que el componente LAN parece ocurrir en respuesta a violaciones morfosintácticas directas (Coulson et al., 1998; Gunter et al., 2000), la obtención de un P600 no requeriría de un error 
sintáctico, pudiendo ocurrir también en oraciones sintácticamente complejas (Friederici et al., 2002) o ambiguas (Osterhout et al., 1994; Frisch et al., 2002). En la actualidad, parece haber un amplio consenso en atribuir el efecto P600 al procesamiento sintáctico, ya que se ha demostrado que es provocado por violaciones sintácticas y por oraciones sintácticamente ambiguas. También se ha demostrado que este efecto está modulado por la complejidad sintáctica. Se supone, de esta manera, que el P600 es un índice de los procesos de integración sintáctica, viéndose la amplitud del P600 afectada por la competencia entre las opciones de integración.

Otros estudios han demostrado que el P600 puede darse o coexistir tanto con el N400 (Frisch y Schlesewsk, 2001) como con el LAN (Coulson et al., 1998; Gunter et al., 2000). Así, el componente LAN se ha relacionado frecuentemente con el procesamiento morfosintáctico (Friederici et al., 2002), mientras que el $\mathrm{P} 600$ con un procesamiento sintáctico posterior, de "re-análisis" (Osterhout et al., 1994; Gunter et al., 2000), o con un procesamiento de la complejidad sintáctica (Kaan et al., 2000).

\subsubsection{AdQUiSición DEL LENGUAJE}

Un campo de gran proyección en neurociencia es el uso los ERP de tipo auditivo para comprender el desarrollo auditivo temprano entre los bebés, ya que permite la recopilación de una cantidad relativamente grande de datos en poco tiempo con un carácter nada invasivo y relativamente fácil de usar, ya que no requiere de la colaboración muy activa por parte de los niños. Normalmente, los estudios que han investigado el desarrollo de ERP auditivos en la infancia, han utilizado principalmente sonidos únicos y simples como estímulos. Sin embargo, en la vida real, los bebés deben decodificar eventos acústicos sucesivos y más complejos en lugar de estímulos auditivos individuales. Con esto en mente, Chen et al. (2016) investigó los ERP auditivos en bebés de 4, 8 y 12 meses con melodias musicales que comprendian tres notas de piano y examinó los ERP que elicitaba cada nota individual en la melodia. Los resultados mostraron que las respuestas de los ERP al inicio de cada nota, es decir, los componentes P1 y N2 infantiles, fueron fácilmente identificables en cada tramo de edad, demostrando, en primer lugar, que el cerebro infantil puede registrar eventos sucesivos acústicos en una secuencia, y, en segundo lugar, que los componentes de los ERP se vuelven más evidentes respecto al inicio en el tiempo para cada evento o estímulo a medida que aumentaba la edad. Además, este estudio también determinó que los bebés más pequeños pueden tener dificultades para responder a aquellos estímulos auditivos que llegan retrasados o tarde en una secuencia estimular, y que esta respuesta de inicio a los eventos tardios podría superponerse a la respuesta incompleta a los estímulos auditivos anteriores.

En las diferentes investigaciones relacionadas con la adquisición del lenguaje destacan, por su relevancia, aquellos trabajos relacionados con la adquisición del propio nombre o el uso del nombre propio en bebés como señal 
social para guiar la atención en el mundo en que se desenvuelven. Hoy en dia sabemos, en parte gracias a trabajos que usan ERP, que los bebés son sensibles a su propio nombre a los 4 meses de edad, aunque todavía existe cierta discrepancia sobre si los bebés usan sus nombres como una señal social. En el trabajo de Parise et al. (2010) con niños de 4-5 meses, el registro de los ERP en respuesta a los nombres reveló que los bebés son capaces de diferenciar su propio nombre de los nombres extraños ya en el primer fonema. Se pudo observar una modulación temprana de la actividad cerebral medida con ERP a nivel frontal, lo que se relaciona con la atención al estímulo, y una más tardía a nivel parietal, lo que a su vez se relaciona con la categorización/codificación del estímulo o nombre. Además, la amplitud de los ERP en respuesta a la percepción de objetos indicaba que los bebés atendian más a los objetos después de escuchar los nombres que los identifican que cuando escuchaban un nombre no relacionado con el objeto percibido. Por lo tanto, a los 5 meses de edad, los bebés no son solo capaces de detectar e identificar su propio nombre, sino que también utilizan los nombres para establecer la relevancia de la información sobre el mundo con el que interaccionan.

Sabemos que los bebés aprenden su lengua materna rápidamente y sin esfuerzo durante los primeros años de vida, en función de la aportación lingüística en su entorno. En estudios conductuales se ha podido demostrar que existiría una discriminación de contornos melódicos y de patrones de acento silábico ya en niños con 1 a 4 meses de edad, constatándose además mediante otras medidas conductuales y cerebrales que existiría una discriminación específica de los contrastes fonéticos a esa edad. Existe cierta controversia sobre si dicha discriminación es específica para cada idioma ya en edad tan temprana, o si esta especificidad por idioma solo se alcanzaria entre los 6 y 12 meses. El estudio de Friederici et al. (2007) demostró, en cualquier caso, que la discriminación específica para cada idioma de los patrones de acentuación silábica puede ya ocurrir en bebés de 4 meses. Usando una tarea de lenguaje tipo Oddball en una muestra de bebés alemanes y franceses, y comparando el procesamiento de palabras disilábicas que difieren en su estructura acentual, e.g, palabras alemanas que se acentúan en la primera sílaba (e.g., pápa), y palabras francesas que se acentúan en la segunda sílaba (e.g., papá), los ERP revelaron que la experiencia con el alemán y el francés afecta de manera diferencial las respuestas cerebrales de estos bebés. Esto es, ya en edad tan temprana la exposición al medio específico de una lengua ofrece una ventaja de procesamiento para la estructura acentual tipica de dicha lengua. Estos datos parecen apuntar, por tanto, a que las representaciones neurales específicas del lenguaje con relación a las formas que tienen las palabras en una lengua nativa o primera lengua pueden estar en el cerebro infantil ya desde los 4 meses de edad.

En otro orden de cosas, son muchos los estudios que han investigado los mecanismos cerebrales implicados en la adquisición y aprendizaje de segunda lengua (Morgan-Short, 2014). Las investigaciones longitudinales sobre el aprendizaje de segunda lengua mediante ERP han demostrado que el 
procesamiento neurocognitivo de la misma cambia cualitativamente con el tiempo. Además, se ha demostrado que en estudiantes de segunda lengua se pueden observar efectos sobre los ERP similares a los hallados en los nativos, en concreto, para aquellas características gramaticales de la segunda lengua que están también presentes en su lengua materna, pero también para las características que, aunque están presentes en su lengua materna, se realizan de manera diferente en la segunda lengua. Por otra parte, nuevas investigaciones han revelado que las diferencias individuales en los ERP pueden explicarse tanto por factores lingüísticos como no lingüísticos. Estudios empíricos recientes han demostrado igualmente que los contextos de entrenamiento o aprendizaje explicitos e implicitos pueden conducir a efectos sobre los componentes de los ERP similares a los nativos en altos niveles de competencia, aunque los contextos implícitos pueden conducir al desarrollo de un estilo más completo de procesamiento similar al nativo, al menos, para el procesamiento sintáctico (Morgan-Short, 2014).

\subsubsection{OTROS ASPECTOS DEL LENGUAJE}

Más allá de estudiar los componentes de los ERP como marcadores cerebrales sobre los desajustes semánticos y sintácticos en el procesamiento del lenguaje, o sobre aquellas variables que más influyen en la adquisición del lenguaje o en el aprendizaje de una segunda lengua, existen también otros estudios que han investigado aspectos particulares relacionados con el uso del lenguaje para determinados efectos retóricos o como instrumento de nuestra capacidad de raciocinio. Así, por ejemplo, gracias a los trabajos con ERP podemos comprender mejor el procesamiento que hacemos a la hora de comprender metáforas convencionales y novedosas, o las respuestas de los sujetos que realizan un razonamiento silogístico.

Con relación al procesamiento complejo de las metáforas, diferentes trabajos han demostrado una línea de conclusiones común: cuando las metáforas nuevas son desconocidas, son también más difíciles de interpretar. Lai et al. (2009) compararon los diferentes ERP que se generaban por la misma palabra objetivo cuando ésta se usó al final de oraciones anómalas, expresiones metafóricas novedosas, expresiones metafóricas convencionales, y expresiones literales. Los resultados mostraron que la amplitud del componente N400 (320-440 ms) fue más negativa (incrementada) para las oraciones anómalas, las metáforas novedosas y las metáforas convencionales, que para las oraciones literales. Sin embargo, en un procesamiento más tardio (440-560 ms), los ERP asociados con metáforas convencionales convergieron con los ERP asociados a las oraciones literales, mientras que los ERP de las metáforas novedosas convergieron con los de las oraciones anómalas.

En relación con los silogismos, y de acuerdo con las medidas conductuales, somos propensos a considerar válida la conclusión de un argumento basándonos en la veracidad de la conclusión en sí misma, más que en la lógica formal del argumento. En un reciente estudio con ERP, RodríguezGómez et al. (2018) encontraron que habia un incremento del componente 
para el procesamiento de las condiciones que incluian conclusiones inválidas precedidas por premisas verdaderas (e.g., "Todos los hombres son mortales"). Por el contrario, cuando las premisas iniciales consistian en declaraciones con prejuicios sociales previamente calificados como falsos (e.g., "Todas las chicas rubias son tontas"), las conclusiones válidas, en lugar de las inválidas, incrementaron la modulación del N400. Teniendo en cuenta cuáles son las situaciones en que podemos modular el componente N400 (i.e., procesos de anticipación de palabras o la integración del significado de una palabra en la representación general de su contexto de uso), estos datos parecen sugerir que las personas no pueden seguir la lógica de un argumento para anticipar los próximos juicios si estos chocan con la veracidad.

\subsubsection{El ESTUdiO DEL LENGUAJE CON ERP EN POBLACIÓN CLÍNICA}

Muchos de los estudios en los que se emplea metodología de ERP se han centrado en estudiar cómo la especialización e integridad de ciertas áreas cerebrales, como las áreas visuales, son cruciales para el procesamiento rápido de palabras leídas, y desempeñan un papel crucial en la adquisición de habilidades de lectura. La desregulación de estas áreas puede estar entre los déficits subyacentes, por ejemplo, a la dislexia del desarrollo. A este respecto, Fraga-González et al. (2017) examinaron en niños disléxicos entre 89 años la especificidad de la activación asociada a palabras al comparar componentes tempranos de los ERP provocados por palabras presentadas visualmente o en cadenas de símbolos sin letras. Los resultados mostraron que el componente N1 se encontraba más pronunciado para las palabras en comparación con los símbolos para ambos grupos. El grupo disléxico, en comparación con el grupo de lectores típicos, sin embargo, mostró unas respuestas del componente N1 más grandes y específicas para las palabras, particularmente lateralizadas al hemisferio izquierdo. Además, se encontraron correlaciones positivas entre las amplitudes N1 y la fluidez de lectura en el grupo disléxico. Estos datos apoyan la noción del componente N1 como un índice sensible al procesamiento visual de palabras e involucrado en la fluidez de lectura, y sugieren que existiria un déficit a nivel de especialización de palabras visuales en niños disléxicos, los cuales deberían seguirse a nivel longitudinalmente para valorar si este déficit aparente en la especialización de palabras visuales disminuye cuando alcanzan niveles más altos de fluidez de lectura.

Como hemos podido observar, una de las ventajas del uso de los ERP es su posibilidad de ser usada en población infantil y por extensión, en individuos pocos colaboradores, por lo que ha sido una herramienta fundamental para estudiar diferentes aspectos cognitivos en individuos con autismo infantil. En el autismo, las anomalias graves en el comportamiento social coexisten con una atención desestructurada y un lenguaje deficiente, siendo la atención a las personas y a los estímulos socialmente relevantes los que se ven más afectados. Debido a que los eventos relacionados con estímulos 
socialmente significativos o con el lenguaje humano son fisicamente complejos, el uso de ERP durante la ejecución de tareas cognitivas es una herramienta de enorme utilidad para explorar aspectos del funcionamiento cerebral que dificilmente pueden ser investigados con otras técnicas en el autismo infantil. En general, se ha sugerido que, en el autismo, existiria una deficiencia en el procesamiento sensorial de aquellos estímulos complejos que contribuiría a los déficits de atención y del lenguaje frecuentemente documentados en esta población. En un estudio paradigmático de Ceponiene et al. (2003) en niños con autismo de alta funcionalidad utilizando ERP, se examinó el procesamiento sensorial y en las primeras fases del proceso atencional a sonidos de diferentes complejidades. Para ello, el referido estudio empleó tonos combinados acústicamente de tipo simples, complejos y vocales, presentados en secuencias separadas en una tarea tipo Oddball. Los resultados relacionados con las respuestas sensoriales mostraron que podian identificarse aquellos componentes de los ERP relacionados, en concreto, con la discriminación automática al cambio de sonido, lo que se manifiesta en el componente MMN, y con la orientación atencional, a la que resulta sensible el componente P3 (frontal). Ello nos estaría indicando que el procesamiento de los sonidos a nivel sensorial se encuentra intacto en los niños con autismo de alta funcionalidad, y que no se ven afectados por la complejidad del sonido o aquellos aspectos relacionados con el "habla". Por el contrario, la orientación involuntaria sí parece que podría verse afectada por la naturaleza del estímulo. En concreto, la orientación (medida con el componente P3 en regiones frontales) era normal respecto a los cambios de los tonos simples y complejos, pero estaba completamente suprimida respecto a los cambios de los estímulos basados en vocales. Estos hallazgos nos demuestran que, en primer lugar, los déficits relacionados con la orientación auditiva en el autismo no pueden explicarse por déficits sensoriales $y$, en segundo lugar, que los déficits de orientación en el autismo podrían ser específicos a los sonidos relacionados con el habla humana.

La investigación de alteraciones del lenguaje mediante ERP suele también ser muy útil en pacientes afectados por distintas afectaciones neurológicas en las que este se ve afectado, siendo frecuente en pacientes afásicos tras haber sufrido un ictus o una cirugía cerebral, o en aquellas dolencias en que se ven afectados aspectos motores de la producción del lenguaje, como ocurre en algunas distonías o en la enfermedad de Parkinson. Es por ello que está cobrando gran interés el estudio de las alteraciones del lenguaje a nivel motor o a nivel de aspectos del procesamiento emocional. Estudios previos han documentado el posible papel de estructuras subcorticales como el estriado en la percepción emocional de la prosodia, particularmente en etapas tempranas del dicho procesamiento, lo que seguramente se deba a un mecanismo de tipo ascendente que modularia la respuesta temporal superior a la prosodia emocional. Además, se cree que el estriado del hemisferio izquierdo no jugaría un papel tan esencial en la detección temprana de la prosodia emocional en comparación con el estriado derecho, que sí podría 
estar más involucrado en este proceso. Es este sentido, el trabajo de Garrido-Vásquez et al. (2013) investigó mediante ERP y una tarea con palabras las alteraciones para detectar la saliencia emocional que tenian pacientes de Parkinson con disfunción principalmente del hemisferio derecho en comparación con otros del hemisferio izquierdo. Los resultados de los ERP mostraron que los pacientes de Parkinson con alteraciones del hemisferio derecho presentaban un léxico predominantemente afectado, mientras que el procesamiento del pseudo-discurso estaba en gran parte intacto en estos mismos sujetos. La amplitud de componente P2 de los ERP se correlacionó también significativamente con las puntuaciones motoras izquierdas y los indices de asimetria, unos datos que sugieren que la detección de saliencia emocional de la prosodia se ve afectada por la degeneración neuronal asimétrica en la enfermedad de Parkinson.

\section{ESTIMULACIÓN CEREBRAL NO INVASIVA AL ESTUDIO DEL LENGUAJE}

\subsection{ESTIMULACIÓN CEREBRAL NO INVASIVA}

Con el advenimiento del uso de técnicas de estimulación cerebral no invasiva, podemos decir que ha comenzado una nueva década en el estudio del lenguaje. Estas técnicas están contribuyendo cada vez más a un mejor entendimiento de la neurofisiología del lenguaje, de una manera como no se había hecho desde los descubrimientos de Broca o Wernicke, arrojando luz sobre los mecanismos de plasticidad tanto en el cerebro sano como en los pacientes que sufren de afasia y otras patologías. Entre las técnicas de estimulación cerebral no invasiva, destacan por su relativa facilidad de uso la estimulación magnética transcraneal (transcranial magnetic stimulation, TMS) y la estimulación transcraneal por corriente continua (transcranial direct-current stimulation, tDCS).

\subsection{ESTIMULANDO EL LENGUAJE: ESTIMULACIÓN TRANSCRANEAL POR CORRIENTE CONTINUA (TDCS)}

En las décadas de 1950 y 1960, y basándose en los primeros experimentos de Galvani sobre bioelectricidad en el siglo XVIII, la tDCS se comenzó utilizando principalmente en investigación con animales. Estos estudios demostraron que los efectos de tDCS se basan principalmente en una modulación del potencial de membrana en reposo (Nitsche y Paulus, 2000). Recientemente, la tDCS fue reintroducida como un medio para inducir plasticidad en el cerebro humano sano en 2000 por Nitsche y Paulus (2000). En los últimos años, esta técnica ha comenzado a utilizarse en el estudio de las funciones motoras y cognitivas para modular la excitabilidad cortical durante largos períodos de tiempo en sujetos sanos y pacientes con lesiones cerebrales. Su mecanismo de acción se basa en la aplicación de una débil corriente eléctrica (entre 1-2 mA) durante 5-30 min sobre el cuero cabelludo 
a través de dos electrodos de superficie (un ánodo y un cátodo). Esta corriente continua pasa a través del tejido intermedio y del cráneo, y gran parte de ella llega también al cerebro cambiando la carga eléctrica en reposo, particularmente debajo del cátodo. La tDCS altera la excitabilidad neuronal cambiando el potencial de membrana de las neuronas superficiales tanto despolarizando como hiperpolarizándola. Dichos efectos duran al menos durante 1 hora tras un único periodo de estimulación. Varios estudios sobre la función motora y cognitiva han documentado que los cambios en el rendimiento y a nivel conductual inducidos por tDCS pueden llegar a persistir incluso más allá del período de estimulación, hasta 6-12 meses después de la intervención (Reis et al., 2009; Cohen-Kadosh et al., 2010).

A nivel neurológico, la tDCS es hoy en dia un frecuente tratamiento rehabilitador con efectos muy positivos en pacientes con ictus, dolor crónico, migraña, fibromialgia, depresión y epilepsia, entre otros. Su uso, además, está permitido en niños de mediana edad, y también como tratamiento para "mejorar" algunas habilidades cognitivas en niños con trastorno de déficit de atención por hiperactividad, donde ha mostrado importantes resultados cuando se aplica conjuntamente con terapia conductual y farmacológica.

\subsubsection{EL USO DE LA TDCS EN EL ESTUDIO DEL LENGUAJE}

La evidencia actual de los estudios que investigan el lenguaje usando tDCS, y apoyados en trabajos que lo combinan con otras técnicas de neuroimagen, muestra que la aplicación de la tDCS induce cambios de la actividad cerebral en redes a gran escala relacionadas con el procesamiento del lenguaje. Del mismo modo, se ha sugerido que la tDCS (anodal) podría modular las oscilaciones cerebrales endógenas de baja frecuencia que no están únicamente restringidas al área seleccionada de estimulación, sino que también dicha modulación se extendería a áreas cerebrales funcionalmente conectadas (Meinzer et al., 2013).

La mayoria de los estudios con tDCS sobre el lenguaje en personas sanas han aplicado la tDCS anodal sobre regiones como el giro frontal inferior (IFG) o el giro temporal superior (STG) del hemisferio izquierdo para potenciar el rendimiento en tareas de aprendizaje o de asignación de nombres a imágenes. El potencial en el uso de la tDCS para mejorar el funcionamiento del lenguaje tiene su mayor exponente cuando se aplica en programas de rehabilitación. La mejora del rendimiento en tareas de lenguaje o en la producción del lenguaje natural ha sido un resultado frecuentemente hallado tras aplicar tDCS anodal.

Por ejemplo, De Vries et al. (2010) estimularon a sujetos sanos el área a IFG izquierda con tDCS (1 mA) durante la fase de adquisición de una tarea de aprendizaje gramatical implícita. Ello provocó una mejora significativa del rendimiento que tuvieron posteriormente los participantes cuando realizaron una tarea de decisión gramatical después de una sola sesión de 20 minutos de tDCS anodal sobre el IFG izquierdo en comparación con tDCS sham (placebo, o simulada) y con tDCS anodal sobre el IFG derecho. Dicha 
mejora estuvo particularmente marcada en la detección de violaciones sintácticas tras recibir tDCS anodal, lo que tiene gran interés para la recuperación de algunos pacientes con afasia tras sufrir un ictus.

Cattaneo et al. (2011) investigaron los cambios inducidos por tDCS anodal en las tareas de fluidez verbal, evaluando la generación de palabras clave tanto de letras como de categorias. Cuando aplicaron tDCS anodal sobre el área de Broca (hemisferio izquierdo), la fluidez fonética y semántica mejoraron, pero cuando estimularon las zonas homólogas del hemisferio derecho permanecieron sin cambios. Ello llevó a estos investigadores a concluir que la fluidez verbal depende de manera crítica del área de Broca y que los estudios de tDCS diseñados para mejorar las funciones del lenguaje en pacientes con déficit de producción de lenguaje oral deberian enfocarse en esta área.

En otro estudio, Ross et al. (2011) investigaron cómo la tDCS influye en la capacidad de usar nombres de personas y lugares famosos. Los resultados mostraron que, cuando los nombres eran conocidos, pero dicho nombre era dificil de recordar, la tDCS aplicada sobre el lóbulo temporal anterior derecho ayudó a aumentar la precisión al nombrar a personas famosas, pero no tuvo ningún efecto sobre la precisión a la hora de nombrar los puntos o lugares de referencia. Este efecto selectivo de precisión para nombrar a personas famosas parece subrayar el papel prominente del lóbulo temporal anterior derecho en la designación adecuada de los estímulos sociales.

Sobre la base del supuesto de que la capacidad de aprendizaje verbal es crucial para adquirir nuevos idiomas en individuos sanos y para la readquisición del lenguaje después de lesiones cerebrales, Flöel et al. (2008) usaron tDCS (20 min, 1mA) sobre el área de Wernicke durante una tarea que implica aprender un tipo de léxico nuevo. Encontraron que la velocidad de aprendizaje y la precisión en general de las respuestas aumentaron con tDCS anodal. Además, la tDCS anodal mejoró el rendimiento significativamente más que el tDCS catodal y que la tDCS sham (simulada), mientras no hubo cambios relacionado con el estado de ánimo, los tiempos de reacción, o el estilo de respuesta. Estos hallazgos sugieren, por tanto, que la tDCS, en combinación con otros entrenamientos intensivos, podría facilitar el aprendizaje verbal y, por lo tanto, podria ser una herramienta de gran utilidad para mejorar la recuperación del lenguaje en los pacientes afásicos.

En este sentido, Monti et al. (2008) fueron de los primeros investigadores en tratar específicamente de aclarar los efectos del tDCS en pacientes con afasia. Aplicando tDCS sobre áreas afectadas frontotemporales del hemisferio izquierdo en pacientes con afasia crónica no fluyentes, evaluaron el efecto que tenía la tDCS de tipo anodal, catodal y sham. Los pacientes fueron evaluados antes e inmediatamente después de tDCS usando una tarea de denominación de imágenes. Después de la estimulación catódica, la precisión en las habilidades de denominación mejoró un 34\%, mientras que en las otras condiciones (tDCS anodal y sham) no hubo una mejoría de las capacidades de denominación, ni tampoco la aplicación a modo de control de la tDCS catodal sobre el área occipital facilitó en ningún caso la tarea de 
denominación. Por lo tanto, en este tipo de pacientes afásicos, la mejoría en la denominación después del tDCS catodal fue dependiente tanto de la polaridad como del área específica estimulada. Debido a que la tDCS catódica parece disminuir la excitabilidad en los circuitos inhibitorios corticales (Lang et al., 2004), la mejora podría estar reflejando una decremento o depresión de la inhibición de las interneuronas corticales inducida por tDCS, lo que en última instancia conduce a la desinhibición y, en consecuencia, a mejorar la función en las áreas del lenguaje dañadas de la corteza cerebral. Aunque aún existe cierto debate sobre el mecanismo específico de la tDCS, este primer trabajo piloto abrió el camino a nuevos estudios sobre cómo se puede usar la tDCS para mejorar el lenguaje en pacientes que presentan alteraciones de esta capacidad.

Debido a que el uso de ls tDCS está permitido en población infantil, son muchos los estudios que lo han usado en niños y adolescentes con trastorno de hiperactividad por déficit de atención o con problemas de dislexia. En niños con dislexia, por ejemplo, son numerosos los estudios que han documentado una mejora de la lectura después del tratamiento con tDCS. Recientemente, Costanzo et al. (2016) trataron de investigar los efectos que la tDCS tiene sobre la lectura y otras habilidades relacionadas con la lectura en niños y adolescentes con dislexia. En concreto, se realizaron diferentes tareas relacionadas con la lectura (lectura de palabras, no palabras y textos; decisión léxica; combinación fonológica; memoria de trabajo verbal; denominación automatizada rápida) en una condición inicial sin tDCS y en otra después de 20 minutos de exposición a tres condiciones diferentes de tDCS: tDCS anodal izquierdo (catodal derecho para mejorar la lateralización izquierda de la región parieto-temporal; tDCS anodal derecho (catodal izquierdo para mejorar la lateralización derecha de la región parieto-temporal); y tDCS sham.

En la lectura de textos, el grupo con la tDCS activa mostró menos errores de lectura tanto en aquellas palabras de baja frecuencia como en tiempos de lectura en general. Estos efectos positivos fueron estables incluso transcurrido un mes después del final del tratamiento, sin que se informara de efectos adversos. Estos hallazgos, bastante novedosos e impactantes, estarían indicando que una sesión de tDCS es capaz de modular algunos aspectos del rendimiento en la lectura de niños y adolescentes con dislexia, destacando además que el efecto depende de la polaridad de la propia estimulación. Estos resultados tras emplear una sola sesión respaldan, por tanto, el papel potencial de la tDCS para desarrollar protocolos de tratamiento, y sugieren los posibles parámetros para la personalización del tratamiento de la tDCS en niños y adolescentes con dislexia.

\subsection{Estimulando EL LENGUAJE: ESTIMULACIÓN MAGNÉtica TRANSCRANEAL (TMS)}

La TMS fue introducida como una técnica no invasiva para la estimulación eléctrica de la corteza humana en la década de 1980 (Barker et al., 
1985). Al investigar las funciones cerebrales mediante TMS, debe tenerse en cuenta que los efectos de TMS dependen criticamente, entre otros, de los parámetros de estimulación, el área o áreas corticales específicas, la tarea empleada y el momento de la estimulación (Siebner et al., 2009).

A nivel fisiológico, un pulso único de TMS provoca la estimulación electromagnética de los axones neuronales, particularmente en las regiones superficiales de la corteza cerebral. La TMS interactúa directa y no invasivamente con el procesamiento cortical al pasar una fuerte y breve corriente eléctrica a través de una bobina de estimulación, que induce a su vez un campo magnético perpendicular, que varía en el tiempo, y que penetra el cuero cabelludo sin atenuación. Este campo magnético induce igualmente una débil corriente de corta duración en el lugar de estimulación que puede excitar o inhibir temporalmente el área estimulada (Bestmann, 2008; Pascual-Leone et al., 2000). Generalmente pulsos de baja frecuencia $(<1 \mathrm{~Hz})$ suelen inhibir mientras que pulsos de alta frecuencia ( $>5 \mathrm{~Hz}$ ) suelen tener efectos excitatorios sobre el área estimulada. La inducción de un campo magnético causa despolarización o disparo de las células nerviosas cerebrales. Cuando se aplican "trenes" repetitivos de pulsos, los disparos repetidos de las neuronas en el tiempo parecen cambiar su actividad intrínseca. El término "estimulación magnética" podría parecer engañoso ya que el fuerte campo magnético variable en el tiempo que se induce en la bobina TMS solo se usa como un medio para generar un campo eléctrico en el cerebro. La mayoría de los estudios han investigado los mecanismos fisiológicos de la TMS en el sistema motor humano porque sus efectos pueden cuantificarse fácilmente registrando el potencial evocado motor (generalmente en la mano o brazo) inducido por TMS como una medida de la excitabilidad neuronal (Pascual-Leone et al., 2000). Cuando se aplica sobre la corteza motora primaria, la TMS puede despolarizar las neuronas del tracto corticoespinal y provocar movimientos contralaterales del músculo de la mano. El tamaño de estos potenciales evocados motores refleja la excitabilidad del sistema corticoespinal (Bestmann, 2008). Para otras regiones del cerebro, tales medidas directas son más dificiles de obtener. A este respecto, los efectos inducidos por la TMS sobre las funciones cognitivas, como el lenguaje, generalmente se cuantifican como cambios en el rendimiento conductual (es decir, la velocidad y precisión de una tarea específica) o cambios en la activación neural.

Una revisión de la literatura indica que el uso de la TMS puede ayudar a aumentar y mejorar el rendimiento cognitivo. Se incluyen aquí las habilidades visuo-espaciales, atención sostenida, memoria de trabajo y episódica, rotación mental, aprendizaje motor, etc (Siebner et al., 2009; Nevler y Ash, 2015). La TMS se puede utilizar también para estimular de forma no invasiva una región cortical específica e interrumpir transitoriamente el procesamiento de información. Estos estudios de "lesión virtual" ofrecen, no solo la capacidad de explorar las relaciones causales entre las regiones cerebrales y las funciones del lenguaje ausentes en la neuroimagen funcional, sino 
también la precisión espacial y temporal que generalmente no está disponible en los estudios de pacientes (Pascual-Leone et al., 2000).

\subsubsection{EL USO DE LA TMS EN EL ESTUdIO DEL LENGUAJE}

Por ejemplo, la TMS se ha utilizado para demostrar subregiones funcionalmente distintas del giro frontal inferior izquierdo, aclarar la relación entre la organización del lenguaje premórbido y la susceptibilidad a lesiones unilaterales, e investigar la contribución de las áreas del lenguaje del hemisferio izquierdo y derecho en la recuperación de la afasia (Cattaneo, 2013). Cuando la TMS se usa como una medida de conectividad funcional, muestra un vínculo estrecho entre palabras de acción y programas motores, apunta a un posible vínculo evolutivo entre los gestos con las manos y el lenguaje, y sugiere un papel en la percepción del habla para el sistema motor subyacente a la producción del habla. En combinación con neuroimagen funcional, puede dilucidar los circuitos responsables de esta participación. Finalmente, TMS incluso puede ser útil para mejorar la recuperación en pacientes afásicos. En otras palabras, el TMS es ya una herramienta importante para estudiar el lenguaje tanto a nivel cognitivo como neuronal, y está claro que los desarrollos adicionales en la metodología TMS probablemente nos llevarán a un mayor avance en el estudio del lenguaje (Schlaug et al., 2011; Cattaneo, 2013).

Gracias a la TMS se ha podido comprender mucho mejor el papel que el área IFG (área de Broca) y del área STG (área de Wernicke) desempeñan en el lenguaje tanto en personas sanas como con alguna alteración neurológica. Así, por ejemplo, Hartwigsen et al. (2010) examinaron la relevancia funcional del IFG posterior derecho en las decisiones fonológicas auditivas y visuales en personas sanas diestras mientras emitian juicios fonológicos y semánticos sobre un mismo conjunto de palabras presentadas auditiva y visualmente mientras recibian TMS sobre el IFG posterior izquierdo, derecho o bilateral. En comparación con la TMS aplicada sobre el IFG anterior, la TMS sobre el IFG posterior perjudicó a los tiempos de reacción y la precisión de las decisiones fonológicas, pero no a las semánticas para las palabras presentadas tanto de forma visual y auditiva. Por su parte, la TMS sobre el IFG izquierdo, derecho o bilateral interrumpió, en un grado similar, el procesamiento fonológico. Estos hallazgos indican que se hace necesario el funcionamiento adecuado del IFG posterior derecho para tomar decisiones fonológicas precisas y eficientes, sin que haya evidencia de que el IFG izquierdo o derecho puedan compensarse entre sí.

El uso preciso de la TMS puede ayudarnos también a delimitar las subdivisiones funcionales que puede tener una misma área cortical. En relación con el estudio del lenguaje, Gough et al. (2005) han intentado demostrar usando TMS si la corteza frontal inferior izquierda anterior (i.e. IFG, área de Broca) se comporta como una sola región o puede, por el contrario, subdivirse en áreas distintas cuando se procesa el significado y el sonido de las 
palabras. Asi, la TMS aplicada sobre la corteza frontal inferior izquierda anterior aumentó selectivamente las latencias de las respuestas cuando los participantes se enfocaron en el significado de las palabras presentadas simultáneamente (i.e., juicios con sinónimos) pero no cuando se enfocaron en el patrón de sonido de las palabras (i.e., juicios homófonos). En contraste, la disociación opuesta se observó en la corteza frontal inferior izquierda posterior, donde la estimulación interfirió selectivamente con la tarea fonológica pero no con la semántica. Esta doble disociación muestra subdivisiones funcionalmente distintas la corteza frontal inferior izquierda anterior que pueden entenderse en términos de conexiones cortico-corticales separables que unen esta región anterior con regiones del polo temporal asociadas con la memoria semántica, mientras que la corteza frontal inferior izquierda posterior estaría conectada funcionalmente con regiones temporo-parietales involucradas en el procesamiento auditivo del lenguaje.

Además de los numerosos estudios sobre el lenguaje o sobre la mejora de la función cognitiva, la TMS tiene un gran interés clínico en la rehabilitación del lenguaje aplicada a la recuperación de pacientes afásicos. De esta manera, son numerosos los trabajos que han usado la TMS de forma repetitiva para mejorar el funcionamiento del lenguaje, incluido la denominación, en pacientes con accidente cerebrovascular con secuelas de afasia crónica no fluida.

En particular, se ha propuesto que la TMS ayuda a mejorar significativamente la neurorrehabilitación. La estimulación repetitiva mediante TMS (rTMS) produce efectos duraderos sobre la excitabilidad y cambios en las conexiones sinápticas. Los estudios de caso único sugieren que el rTMS de $1 \mathrm{~Hz}$ en la parte homóloga anterior del área Broca en el hemisferio derecho (pars triangularis) puede inducir la recuperación del lenguaje en la afasia crónica en pacientes tras haber sufrido un accidente cerebrovascular, seguramente al modular la actividad en la red cortical del lenguaje distribuida a nivel bi-hemisférico (Mendoza et al., 2016). En ocasiones, las mejorías sobre la función del lenguaje no ocurren inmediatamente o en las primeras semanas, sino que los resultados relativamente favorables pueden darse varios meses después del tratamiento y, más tarde, particularmente en la designación y descripción de imágenes (Barwood et al., 2013). En un trabajo sobre pacientes con afasia subaguda durante los primeros meses después del accidente cerebrovascular, Thiel et al. (2013) han encontrado que la aplicación de rTMS a $1 \mathrm{~Hz}$ (protocolo de 10 dias, rTMS de $20 \mathrm{~min}$ ) sobre el pars triangularis del hemnisferio derecho mejoró sustancialmente el lenguaje de los pacientes, unos resultados que han llegado a sugerir que el homólogo del área de Broca en el hemisferio derecho podría funcionar sobre la neuroplasticidad eficiente del lenguaje en la afasia subaguda posterior al accidente cerebrovascular (Kapoor, 2017). En otras palabras, aplicando rTMS de $1 \mathrm{~Hz}$, la actividad neuronal en la parte anterior del pars triangularis derecho, que normalmente inhibiria su homólogo izquierdo, se reduce. Esto podría facilitar (o desinhibir) la función neuronal y la reorganización en el hemisferio izquierdo (Hamilton et al., 2011). 


\subsection{INTERPRETACIÓN DE LOS RESULTADOS OBTENIDOS CON TMS/TDCS DESPUÉS DE UNA TAREA DE LENGUAJE (COGNITIVA)}

Es primordial tener en cuenta varias consideraciones importantes a la hora de interpretar los resultados que solemos obtener cuando aplicamos tDCS/TMS en sujetos sanos o en pacientes en experimentos relacionados con la mejoría o rehabilitación del lenguaje.

Los efectos conductuales inducidos tanto por tDCS o TMS pueden ser equívocos o inducir a errores en su interpretación, ya que el rendimiento en una misma tarea cognitiva puede ser facilitado o inhibido por el uso de tDCS/TMS dependiendo del área estimulada. Además, los mismos parámetros de estimulación y lugares "objetivo" donde se aplica pueden tener efectos opuestos dependiendo del tipo de tarea que se esté realizando. Asimismo, los efectos conductuales observados pueden ser el resultado no solo de la estimulación del área objetivo, sino que pueden ser consecuencia de la estimulación indirecta de alguna otra área cerebral diferente al sitio estimulado.

Uno de los grandes desafios al usar técnicas de estimulación no invasiva, hace referencia a la dificultad inherente a la interpretación de un resultado nulo, o, en otras palabras, cuando el rendimiento en una tarea no se ve afectada por el uso de tDCS/TMS sobre un área específica. Ello se puede deber a diferentes factores, como que: i) la estimulación fue insuficiente para afectar el funcionamiento del área objetivo; dos áreas cerebrales participan igualmente en la ejecución de la tarea cognitiva específica, de modo que interferir con solo una de ellas no deriva en un cambio conductual o de rendimiento manifiesto; o iii) existiría una compensación gracias a la cual otra área cerebral asumiria la función respectiva de la región directamente estimulada.

\section{REFERENCIAS}

BARKER, A. T. et al. (1985): "Non-invasive magnetic stimulation of human motor cortex", The Lancet, 325(8437), pp. 1106-1107.

BARWOOD, C. H. et al. (2013): "Long term language recovery subsequent to low frequency rTMS in chronic non-fluent aphasia", NeuroRehabilitation, 32(4), pp. 915-928.

BESTMANN, S. (2008): "The physiological basis of transcranial magnetic stimulation", Trends in Cognitive Sciences, 12(3), pp. 81-83.

BRESSLER, S. L. \& DING, M. (2006): "Event-Related Potentials", Akay, M. (ed.): Wiley Encyclopedia of Biomedical Engineering, New York: John Wiley \& Sons, Inc., pp. 1-8.
CARRETIÉ, L. \& IGLESIAS, J. (2008): Psicofisiologia: Fundamentos metodológicos, Madrid: Pirámide.

CATTANEO, L. (2013): "Language", Lozano, A. M. \& Hallett, M. (eds.): Handbook of Clinical Neurology, Amsterdam: Elsevier, 116, pp. 681-691.

CATTANEO, Z. et al. (2011): “Transcranial direct current stimulation over Broca's region improves phonemic and semantic fluency in healthy individuals", Neuroscience, 183, pp. 64-70.

CEPONIENE, R. et al. (2003): "Speechsound-selective auditory impairment in children with autism: 
They can perceive but do not attend", Proceedings of the National Academy of Sciences, 100(9), pp. 5567-5572.

CHEN, A. et al. (2016): "Auditory ERP response to successive stimuli in infancy", PeerJ, 4, pp. 1580.

COHEN KADOSH, R. et al. (2010): "Modulating neuronal activity produces specific and long-lasting changes in numerical competence", Current Biology, 20(22), pp. 2016-2020.

COSTANZO, F. et al. (2016): "Reading changes in children and adolescents with dyslexia after transcranial direct current stimulation", Neuroreport, 27(5), pp. 295-300.

COULSON, S. et al. (1998): "Expect the unexpected: Event-related brain response to morphosyntactic violations", Language and Cognitive Processes, 13(1), pp. 21-58.

DE VRIES, M. H. et al. (2010): "Electrical stimulation of Broca's area enhances implicit learning of an artificial grammar", Journal of Cognitive Neuroscience, 22(11), pp. 2427-2436.

FLÖEL, A. et al. (2008): "Noninvasive brain stimulation improves language learning", Journal of Cognitive Neuroscience, 20(8), pp. 14151422.

FRAGA GONZÁLEZ, G. et al. (2017): "Contributions of letter-speech sound learning and visual print tuning to reading improvement: evidence from brain potential and dyslexia training studies", Brain Sciences, 7, pp. 10.

FRIEDERICI, A. D. (2002): "Towards a neural basis of auditory sentence processing", Trends in Cognitive Sciences, 6(2), pp. 78-84.

FRIEDERICI, A. D. et al. (2007): "Brain responses in 4-month-old infants are already language specific", Current Biology, 17(14), pp. 12081211.

FRIEDERICI, A. D. et al. (2002): "Distinct neurophysiological patterns reflecting aspects of syntactic com- plexity and syntactic repair", Journal of Psycholinguistic Research, 31(1), pp. 45-63.

FRIEDERICI, A. D. et al. (1993): "Eventrelated brain potentials during natural speech processing: effects of semantic, morphological and syntactic violations", Cognitive Brain Research, 1(3), pp. 183-192.

FRISCH, S. et al. (2002): "The P600 as an indicator of syntactic ambiguity", Cognition, 85(3), pp. B83-92.

FRISCH, S. \& SCHLESEWSKY, M. (2001): "The N400 reflects problems of thematic hierarchizing", NeuroReport, 12(15), pp. 3391-3394.

GARRIDO-VÁSQUEZ, P. et al. (2013): “An ERP study of vocal emotion processing in asymmetric Parkinson's disease", Social Cognitive and Affective Neuroscience, 8(8), pp. 918927.

GOUGH, P. M. et al. (2005): "Dissociating linguistic processes in the left inferior frontal cortex with transcranial magnetic stimulation", Journal of Neuroscience, 25(35), pp. 8010-8016.

GUNTER, T. C. et al. (2000): "Syntactic gender and semantic expectancy: ERPs reveal early autonomy and late interaction", Journal of Cognitive Neuroscience, 12(4), pp. 556-568.

HAMILTON, R. H. et al. (2011): "Mechanisms of aphasia recovery after stroke and the role of noninvasive brain stimulation", Brain and Language, 118(1-2), pp. 40-50.

HARTWIGSEN, G. et al. (2010): "The right posterior inferior frontal gyrus contributes to phonological word decisions in the healthy brain: Evidence from dual-site TMS", Neuropsychologia, 48(10), pp. 3155-3163.

KAAN, E. et al. (2000): "The P600 as an index of syntactic integration difficulty", Language and Cognitive Processes, 15(2), pp. 159-201.

KAPOOR, A. (2017): "Repetitive transcranial magnetic stimulation therapy for post-stroke non-fluent aphasia: 
A critical review", Topics in Stroke Rehabilitation, 24(7), pp. 547-553.

KUTAS, M. \& FEDERMEIER, K.D. (2000): "Electrophysiology reveals semantic memory use in language comprehension", Trends in Cognitive Sciences, 4 (12), pp. 463-470.

KUTAS, M. \& HILLYARD, S.A. (1984): "Brain potential during reading reflect word expectancy and semantic association", Nature, 307, pp. 161-163.

KUTAS, M. \& HILLYARD, S.A. (1980): "Reading senseless sentences: Brain potentials reflect semantic incongruity", Science, 207 (4427), pp. 203-205.

LAI, V. T. et al. (2009): "Comprehending conventional and novel metaphors: an ERP study", Brain Research, 1284, pp. 145-155.

LANG, N. et al. (2004): "Effects of transcranial direct current stimulation over the human motor cortex on corticospinal and transcallosal excitability", Experimental Brain Research, 156(4), pp. 439-443.

MEINZER, M. et al. (2013): "Anodal transcranial direct current stimulation temporarily reverses age-associated cognitive decline and functional brain activity changes", Journal of Neuroscience, 33(30), pp. 12470-12478.

MENDOZA, J. A. et al. (2016): "Repetitive transcranial magnetic stimulation in aphasia and communication impairment in post-stroke: systematic review of literature", Journal of Neurology \& Translational Neuroscience, 4, pp. 2333-7087.

MONTI, A. et al. (2008): "Improved naming after transcranial direct current stimulation in aphasia", Journal of Neurology, Neurosurgery \& Psychiatry, 79(4), pp. 451-453.

MORGAN-SHORT, K. (2014): "Electrophysiological approaches to understanding second language acquisition: A field reaching its potential", Annual Review of Applied Linguistics, 34, pp. 15-36.
NÄÄTÄNEN, R. (2003): "Mismatch negativity: clinical research and possible application", International Journal of Psychophysiology, 48(2), pp. 179-188.

NEVILLE, H. J. et al. (1991): "Syntactically based sentence processing classes: evidence from event-related brain potentials", Journal of Cognitive Neuroscience, 3(2), pp. 151165.

NEVLER, N. \& ASH, E. L. (2015): “TMS as a Tool for Examining Cognitive Processing", Current Neurology and Neuroscience Reports, 15(8), p. 52.

NIEDERMEYER, E. \& LOPES DA SILVA, F. (1999): Electroencephalography: Basic principles, clinical applications and related fields, Philadelphia: Lippincott Williams \& Wilkins.

NITSCHE, M. A. \& PAULUS, W. (2000): "Excitability changes induced in the human motor cortex by weak transcranial direct current stimulation", The Journal of physiology, 527(3), pp. 633.

OSTERHOUT, L. et al. (1994): "Brain potentials elicited by garden-path sentences: Evidence of the application of verb information during parsing", Journal of Experimental Psychology: Learning, Memory and Cognition, 20(4), pp. 786-803.

PARISE, E. et al. (2010): "Did You Call Me? 5-Month-Old infants own name guides their attention", PLOS One, 5(12), pp. e14208.

PASCUAL-LEONE, A. et al. (2000): "Transcranial magnetic stimulation in cognitive neuroscience-virtual lesion, chronometry, and functional connectivity", Current Opinion in Neurobiology, 10(2), pp. 232-237.

PICTON, T. W. \& HILLYARD, S. A. (1988): "Endogenous event-related potentials", PICTON, T. W. (ed.): Handbook of electroencephalography and clinical neurophysiology (Vol 3). Human event-related potentials, Amsterdam: Elsevier, pp. 361425. 
PIZZAGALLI, D. A. (2007): "Electroencephalography and high-density electrophysiological source localization", Cacioppo, J. T., Tass-Inary, L. G. \& Berntson, G. G. (eds.): Handbook of psychophysiology, Cambridge: Cambridge University Press, pp. 56-84.

POLICH, J. \& HERBST, K. L. (2000): "P300 as a clinical assay: rationale, evaluation, and findings", International Journal of Psychophysiology, 38(1), pp. 3-19.

REIS, J. et al. (2009): "Noninvasive cortical stimulation enhances motor skill acquisition over multiple days through an effect on consolidation", Proceedings of the National Academy of Sciences, 106(5), pp. 1590-1595.

RODRÍGUEZ-GÓMEZ，P. et al. (2018): "When logical conclusions go against beliefs: an ERP study",
Language, Cognition and Neuroscience, 33(6), pp. 687-697.

ROSS, L. A. et al. (2011): "Improved Proper Name Recall in Aging after Electrical Stimulation of the Anterior Temporal Lobes", Frontiers in Aging Neuroscience, 3, pp. 16.

SCHLAUG, G. et al. (2011): "The use of non-invasive brain stimulation techniques to facilitate recovery from post-stroke aphasia", Neuropsychology Review, 21(3), pp. 288301.

SIEBNER, H. R. et al. (2009): "How does transcranial magnetic stimulation modify neuronal activity in the brain? Implications for studies of cognition", Cortex, 45(9), pp. 10351042.

THIEL, A. et al. (2013): "Effects of noninvasive brain stimulation on language networks and recovery in early poststroke aphasia", Stroke, 44(8), pp. 2240-2246. 